dissymétrique de chacun des jeux de balais : le décalage symétrique ne permet pas la même surcharge.

Avec un rhéostat intercalé dans le circuit du stator, on obtiendra, à partir de la plus basse vitesse à vide pour laquelle le moteur est prévu, des caractéristiques analogues à celles du moteur d'induction qui aurait cette plus basse vitesse à vide pour synchronisme.

Ce réglage n'est évidemment pas économique, mais il convient pour réaliser des vitesses de passage. Aucune difficulté de commutation n'apparait dans ce fonctionnement, car la tension au collecteur est indépendante de la vitesse, ce qui n'était pas le cas des machines précédentes.

b) Puissance wattée. Puissance déwattée. - Ce que nous avons dit du moteur shunt à double alimentation s'applique sans modification. Le facteur de puissance au couple normal est voisin de 1 au synchronisme et aux grandes vitesses; au demisynchronisme, il peut atteindre 0,75 avec décalage dissymétrique et ne dépasse guère 0,55 avec décalage symétrique.

Le diagramme de courant a même allure que pour le moteur shunt à double alimentation (fig. 53). Toutefois, la construction générale de la fig. 45 n'est applicable que si l'on néglige la réaction de l'enroulement à collecteur.

c) Démarrage. - Ce que nous avons dit du moteur shunt à double alimentation s'applique ici ; il convient en règle générale de prévoir un rhéostat.
D) Conditions et étendue du réglage de la vitesse. - La raison de commutation fixe une limite à la puissance par pôle, pour un glissement maximum donné au-dessus du synchronisme.

D'autre part, pour la raison de chute de vitesse entre vide et charge, aussi bien au-dessus qu'au-dessous du synchronisme, et du courant à vide exagéré aux basses vitesses, le glissement est limité à $\pm 50 \%$.

Le réglage normal est donc de

$$
\frac{N_{s}}{2} \text { à } \frac{3}{2} N^{s} \text { soit } 1 \text { à } 3 .
$$

L'emploi d'une tension réduite est possible comme pour le moteur série, avec les mêmes avantages pour le cos $\varphi$ et le rendement dans la marche à faible charge.

E) Puissances et vitesses réalisables. - Le moteur peut être construit pour des puissances par pôle de l'ordre de $10 \mathrm{CV}$. Il est nécessairement à basse tension, $500 \mathrm{~V}$. au maximum - du fait de l'alimentation par les bagues.

F) Variation de la tension du réseau. - La vitesse du moteur est peu sensible aux variations de tension du réseau. Dans le cas du réglage de 1 à 3 , la variation relative de vitesse à vide, aux vitesses extrêmes, est de l'ordre du dixième de la variation relative de tension.

(Extrait de Jeumont, avril-juin 1928).
(A suivre.)

\title{
Note sur l'alimentation rationnelle de l'éclairage électrique
}

\author{
par Paul Basiaux
}

\section{VERS LA SUPPRESSION DES POINTES JOURNALIÈRES}

L'utilisation moyenne de l'éclairage électrique est d'environ 1.000 heures par an, soit le $1 / 8$ du temps. On ne peut pas imaginer de régime plus désastreux pour l'amortissement d'un matériel et d'installations dont le prix augmente de jour en jour.

Il est certain qu'au prix actuel de vente en détail de l'énergie d'éclairage, il n'est pas toujours prouvé que le distributeur réalise un bénéfice. Les calculs de rendement financier sont établis sur la masse de l'énergie distribuée et, en général, c'est le client de 2 à 3.000 heures d'utilisation annuelle (force motrice, chauffage, etc.) qui permet d'équilibrer le budget.

Car il faut bien se rendre compte du fait que la partie de l'installation destinée à la pointe ne fonctionne pas pendant toute la durée de l'éclairage, mais seulement pendant le faible moment de chevauchement de l'éclairage et de la force motrice. Cette durée n'est donc pas de 1.000 heures par an mais souvent de moins de 300 heures et encore pas pour le maximum de la puissance installée.

Or, la puissance de pointe atteint souvent de 30 à $50 \%$ du total des installations. Se rend-on bien compte du rendement déplorable d'une installation et d'un matériel dont la majeure partie travaille pendant $1 / 20$ ou même $1 / 30$ du temps ?

Il faut bien remarquer que le calcul ne doit pas porter uniquement sur les groupes supplémentaires mais sur les frais d'établissement de toute la centrale et des chaudières s'il s'agit d'usines à vapeur, et de tous les travaux et organes de dérivation et de distribution d'eau s'il s'agit d'usines hydrauliques. Et encore, pour ces dernières, je suppose l'existence d'un réservoir de régularisation journalière alors qu'il existe encore de nombreuses installations hydrauliques marchant au fil de l'eau sans aucune possibilité d'accumulation.

Pour de nombreuses usines hydrauliques de faible et moyenne puissance alimentant une majorité de clients d'éclairage, le problème est encore plus compliqué.

Encore les groupes producteurs d'énergie et les transformateurs peuvent-ils alimenter successivement divers usagers tandis que les distributeurs d'éclairage ne peuvent servir qu'à un seul objet et pendant un temps très court.

L'organe le plus sensible au point de vue de l'amortissement est donc, le plus souvent, le réseau de distribution. On peut dire, tant pour les lignes à haute et basse tension que pour les postes de transformation, que, en général, $50 \%$ de leur valeur devrait être amortis en tablant sur un travail de une heure sur vingtquatre.

Cette moitié est justement celle qui est exigée par la pointe d'éclairage. Il faut ajouter que le client d'éclairage possède toujours une installation de puissance exagérée ne fonctionnant à pleine charge qu'accidentellement pendant quelques heures par an.

On peut donc affirmer que la distribution d'énergie électrique aux prix pratiqués actuellement est un leurre pour l'exploitant et que seuls les clients de force motrice permettent aux distributeurs de réaliser un bénéfice. 
La clientèle d'éclairage est donc à tous points de vue désastreuse et il est certain qu'on lui vend fort souvent 1 fr. 50 ce qui revient en réalité à 5 francs et peut-être plus. Si l'on ajoute que le client de force motrice emploie souvent plus de $50 \%$ de sa puissance installée, pendant 2.000 heures par an, tandis que le client d'éclairage n'emploie pas le tiers de sa puissance installée pendant 1.000 heures par an, on se rendra compte de la complication du problème à résoudre.

Les tarifs qui exigent des minima proportionnels à la puissance installée n'établissent pas une compensation suffisante. II s'agit bien entendu ici du petit client et du client moyen, c'est-à-dire la grosse majorité.

Je n'envisage pas les grands éclairages réguliers ni les imporlants clients de force motrice qui sont des cas particuliers, Lrailés comme tels du reste par les fournisseurs d'énergie.

Il faut remarquer aussi que rien n'est plus variable que les conditions d'exploitation de différents secteurs électriques, que l'on passe d'une région à une autre, d'un pays au voisin, d'une faible puissance à une puissance considérable. Il est donc impossible d'établir à ce sujet des moyennes ou un semblant de statistique et les chiffres donnés le sont purement à titre d'exemple.

Le client idéal est celui qui possède des appareils de chauffage à accumulation absorbant régulièrement une faible puissance pendant 24 heures par jour.

Cette utilisation qui n'est pas assez encouragée ne jouit pas encore partout de tarifs suffisamment bas.

La science de l'éclairage ne possède pas un organe aussi simple ni aussi robuste que l'accumulateur de chauffage mais cependant l'accumulateur électrique mérite une attention spéciale de la part des distributeurs.

Malgré son rendement qui atteint à peine $50 \%$, son encombrement, son poids et son entretien un peu particulier, cet appareil a rendu de grands services à la distribution électrique à ses débuts, surtout quand il ne s'agissait encore que de clients d'éclairage. L'accumulateur électrique joue encore un rôle honorable en traction.

Bien entendu, lorsque la force motrice a pris de l'extension, la puissance des centrales et des sous-stations a tellement augmenté que les batteries d'accumulateurs auraient dû prendre un développément prohibitif et que leur perte de.rendement aurait enlevè à peu près totalement le bénéfice d'une meilleure utilisation.

Mais si la grosse batterie placée au départ est incapable de donner satisfaction, la petite batterie placée à l'arrivée va nous permettre de résoudre le problème.

\section{Prenons un exemple concret :}

"Un client d'éclairage possédant une installation de 10 lampes de 50 bougies, dont la consommation moyenne journalière correspond à 3 lampes fonctionnant pendant trois heures par jour ou environ 1.000 heures par an."

Ce client a le droit d'allumer ses dix lampes à la fois mais s'il use de ce droit une ou deux fois par an seulement, il force cependant le distributeur à tripler ses installations de production et de distribution. Le client d'éclairage n'a de ce fait presque jamais une utilisation de 1.000 heures puisque la marche normale ne porte que sur le $1 / 3$ de son équipement; il n'est donc en réalité assez souvent qu'un client de 300 heures. (Et encore ici ne lient-on pas compte des heures de pointe qui, par le chevauchement de la force motrice el de l'éclairage, forcent le distributeur à augmenter encore plus sa réserve.)

Installons chez ce client d'éclairage pris comme exemple, une petite batterie d'accumulateurs capable d'alimenter 10 lampes de 50 bougies pendant trois ou quatre heures, batterie qui pourra alimenter trois lampes de 50 bougies pendant trois soirées sans recharge en cas d'interruption accidentelle dans l'alimentation. Cet avantage de sécurité permettant aussi certains arrêts journaliers de la centrale a bien sa valeur.

La présence d'un organe de charge d'accumulateurs permet également d'utiliser des lampes portatives pour la circulation dans les caves, les garages, les écuries, etc...

On s'apercevra du reste rapidement que les nombreux avantages, apportés dans chaque foyer par une petite " masse d'énergie " de réserve, compenseront largement les faibles inconvénients.

La batterie sera chargée pendant 24 heures par jour et débitera pendant trois heures par jour sur 150 bougies (Supposons que les lampes consomment 1 watt par bougie).

Sans batterie, notre client exigeait une puissance instantanée de 500 watts, tandis que s'il possède une batterie, la puissance continue de charge, compte tenu du rendement des appareils convertisseurs et des accumulateurs, sera réduite à 50 watts, soit le dixième de l'ancienne solution.

Cette différence est énorme et mérite bien que l'on examine le problème de plus près. La batterie, légèrement suralimentée, fournira seule les surcharges accidentelles et de peu de durée.

On pourrait également envisager la charge des accumulateurs en dehors des heures d'alimentation de la force motrice, avec commande des interrupteurs au moyen d'un courant de forme spéciale envoyé de la centrale, de même que les divers usages pourraient être alimentés soit simultanément soit alternativement par des courants de natures différentes, mais ces problèmes un peu complexes ne sont pas encore au point.

On objectera à tout ce qui précède que le mauvais rendement des accumulateurs représentera une perte énorme d'énergie, mais à quel chiffre estime-t-on la perte totale dans les distributions au moment de la pointe d'éclairage ? Le plus souvent, cette perte est comparable à celle que réalisent les accumulateurs et si l'on voulait la faire descendre à des chiffres normaux, on arriverait à des poids de cuivre représentant un capital dont le bon tiers ne s'amortirait que pendant quelques heures par an.

On voit de suite l'intérêt qu'il y a à installer les batteries chez le client, c'est-à-dire en bout de ligne et non à la centrale ou même à la sous-station.

Au point de vue du capital à investir et en tenant compte de l'éloignement des centrales par rapport aux centres de distribution, je prétends que la solution des batteries sera souvent plus avantageuse ou en tous cas fera équilibre à l'économie réalisée sur les lignes de transport et de distribution. Quant à l'économie d'équipement réalisée dans les centrales et les sousstations, ce sera l'économie du projet.

Pour les distributeurs d'énergie d'éclairage déjà existant, la charge étant réduite au dixième, les neuf dixièmes libérés de la capacité pourront servir à la distribution d'énergie de chauffage. La réalisation de ce problème si désirable pour le fournisseur et pour l'usager n'a pas été retardée par le mauvais rendement ni par les difficultés de mise au point des appareils d'utilisation mais surtout par l'importance des capitaux à mettre en 
œuvre pour augmenter la capacité des réseaux de distribution. Comme les appareils d'éclairage ne recevront plus que du courant transformé, on pourra porter la tension des distributeurs extérieurs à 220 volts au lieu de 125 , c'est-à-dire avec prises sur les phases sans usage du fil neutre. Les isolements actuels suffisent en général pour passer d'une tension à l'autre sans inconvénient. On voit tout l'intérêt financier de l'énorme développement de la capacité des réseaux qui résultera automatiquement et sans frais de cette modification.

Les batteries et leur matériel de charge et de réglage appartiendront au distributeur qui en fera l'entretien.

L'industrie automobile et la T. S. F. nous ont habitués ces derniers temps aux petites batteries portatives, et, tant pour la charge que pour le réglage el l'entretien, il n'y aura rien à innover.

Il est difficile d'établir à l'heure actuelle des prix définitifs pour les organes de la nouvelle solution, mais par comparaison avec des appareils semblables utilisés actuellement pour l'automobile, on pourrait estimer à 10 francs par mois les frais d'amortissement et d'entretien de la batterie d'un petit client d'éclairage.

Pour cette alimentation qui sera régulière et continue, on établira facilement des prix forfaitaires; le compteur d'éclairage pourra donc être supprimé, d'où nouvelle économie en faveur de la batterie.

Les installations de force motrice et de chauffage resteront naturellement inchangées.

Pour la charge des accumulateurs, le courant du secteur sera transformé par un redresseur, appareil actuellement bien au point.

Deux solutions se présentent pour l'utilisation du courant de la batterie :

10 Utilisation directe en basse tension; batterie de 12 volts, par exemple.

Cette solution nécessitera un changement dans le mode actuel des installations et dans la fabrication des appareils d'utilisation : lampes, petits moteurs des appareils de ménage, etc...

De plus, vu la faible tension et l'intensité plus grande du courant, les frais d'installation intérieure seront plus élevés.

$2^{\circ}$ Transformation du courant de la batterie en un courant ondulé de 125 volts au moyen d'un interrupteur rotatif donnant par exemple 50 interruptions par seconde et d'un transformateur statique. Cette solution permettra d'utiliser une batterie plus simple, de 6 volts par exemple.

Le moteur de l'interrupteur sera composé d'une simple bobine et d'un volant en fer doux à plots, il empruntera une solution connue et extrêmement simple et robuste. On aurait pu penser à l'interrupteur électrolytique de Wehnelt, mais sa sécurité de fonctionnement n'est pas absolue. Cet interrupteur fournit de très hautes fréquences qui peuvent avoir lenr intérèt pour l'alimentation de tubes au néon.

Le petit moteur qui ne sera mis en marche qu'aux heures d'éclairage aura une consommation extrêmement faible. Il est possible de réaliser la mise en marche automalique de cet organe par simple fermeture de l'un quelconque des interrupteurs d'éclairage. On peut aussi envisager l'emploi d'un interrupteur automatique à mercure pour toute l'installation ou pour chaque appareil d'éclairage.

Le grand avantage de cette deuxième solution serait de permettre la récupération de la perte de rendement de la batterie en appliquant le principe de la lumière froide proposé par $F$. Dussaud.

On sait en quoi réside ce principe :

La lampe survoltée consomme par bougie beaucoup moins que la lampe sous-voltée. Mais, pour obtenir une différence de rendement appréciable, il faudrail survolter dans de très grandes proportions, au double par exemple. Or, à cette tension, les filaments ne résislent pas, il s'agit donc de les refroidir. On y réussit en réalisant des interruptions rapides pendant lesquelles les filaments ont le temps de se refroidir sans perdre leur luminosité. L’économie résultante est très sensible.

Pourquoi les essais si intéressants de M. Dussaud ne sont-ils pas sortis du laboratoire ? Vraisemblablement parce qu'il n'était pas possible de les appliquer en grand aux centrales, à cause de la formidable intensité en jeu et à cause aussi de la déformation du nouveau courant qui se prétait moins bien à la force motrice et au chauffage.

Mais la solution proposée dans la présente note, ramenant le problème à la division infinie des distributions d'éclairage, chacune de ces petites distributions étant de très faible intensité et ne s'appliquant qu'à l'éclairage, l'idée de Dussaud semble devoir être remise en lumière.

L'alimentation direcle par ce système présente le danger de destruclion des lampes en cas d'arrêt accidentel de l'interrupteur sur un contact. Cet inconvénient n'existera plus avec la solution proposée, car, pour que le transformateur statique fournisse du courant au secondaire, il faut nécessairement que l'interrupteur qui alimente le primaire soit en marche, puisque cet interrupteur reçoit du courant continu. On évitera le danger des effets violents d'une luminosité trop blanche en employant les systèmes d'éclairage indirect déjà très répandus.

Comme il ne s'agit pas de favoriser uniquement le client en rendant le problème financier du distributeur impossible à cause de la réduction de la consommation, on établira facilement une nouvelle formule de tarification qui sauvegardera les intérêts des deux parties.

Ce qui précède n'est pas une étude de mise au point mais la simple indication d'un principe qui mérite, à mon avis, d'être pris en considération pour le plus grand développement de la distribution et de l'utilisation de l'énergie électrique. 